\title{
So finden Sie den Grund für seinen Frust
}

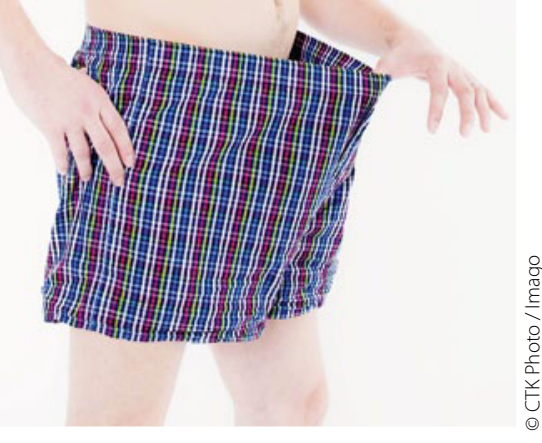

Kranke Seele oder kranker Körper: Woran liegt es, dass nichts mehr geht?

- Eine erektile Dysfunktion (ED) liegt vor, wenn das Aufbauen und Aufrechterhalten einer ausreichenden Erektion für befriedigenden Geschlechtsverkehr nicht möglich ist, entweder in einem Zeitraum von über sechs Monaten oder bei über 70\% der koitalen Versuche.

Obwohl Depression, Versagens- und Erwartungsängste nach wie vor eine gewisse Rolle spielen, sind Stoffwechselerkrankungen wie Diabetes, Hyperlipidämie oder Hypogonadismus als mögliche Ursachen viel bedeutsamer. Als Risikofaktoren kommen Bluthochdruck und die Niereninsuffizienz hinzu. Nicht zuletzt können Medikamente wie Antihypertensiva, Psychopharmaka, Antihistaminika, Lipidsenker oder Analgetika, aber auch der chronische Abusus von Alkohol, Nikotin oder Drogen die Erektionsfähigkeit beeinträchtigen.

Die ED wird heute als Frühwarnsymptom für eine latente koronare Herzkrankheit angesehen. „Männer mit einer ED in jungen Jahren haben ein wesentlich höheres Risiko für Herzinfarkt und Schlaganfall als Männer mit einer guten Sexualfunktion“, erklärte Prof. Ulrich Humke, Stuttgart. Ein außerordentlich hohes Risiko für eine ED haben Diabetiker. $60 \%$ von ihnen sind davon betroffen.

\section{Komplexe Ursachensuche}

Die Suche nach den Ursachen einer ED beginnt mit einer allgemeinen und einer Sexualanamnese. Mithilfe standardisier-

\begin{abstract}
Während die erektile Dysfunktion als die mit Abstand häufigste sexuelle Störung meist erst in der zweiten Lebenshälfte zu einem Thema wird, tritt die Ejaculatio praecox unabhängig vom Alter bei etwa jedem vierten Mann auf. Von der Psyche über Stoffwechselstörungen und Gefäßerkrankungen bis zu Medikamenten - viele Ursachen können dahinter stecken.
\end{abstract}

ter Fragebögen wie dem „International Index of Erectile Function“ (IIEF) lässt sich die Schwere des Problems erfassen. Bei Verdacht auf eine psychogene Problematik sollte der Patient frühzeitig psychologisch mitbetreut werden, erklärte Humke.

Die nicht invasive Diagnostik umfasst das äußere Genitale und sekundäre Geschlechtsmerkmale wie Gynäkomastie, Körperhaar- und Fettverteilung. Danach sollten das Herz-Kreislauf-System und endokrine Veränderungen untersucht werden. Humke empfahl auch, altersabhängig den Gefäßstatus und den neurologischen Status zu bestimmen. Das Labor fokussiert auf mögliche Stoffwechselstörungen. Bei der erweiterten Hormondiagnostik ist das Testosteron der zentrale Parameter. Bei entsprechendem Verdacht sollte auch das PSA zur Abgrenzung des Karzinomrisikos mitbestimmt werden. Als semiinvasive Untersuchung kommt der Schwellkörperinjektionstest (SKIT) infrage. Mit Hilfe der farbkodierten Duplexsonografie lässt sich die Flussgeschwindigkeit der Arteria profunda penis mit und ohne SKIT bestimmen.

\section{Nicht alle Therapieoptionen finden eine hohe Akzeptanz}

Nicht bei jeder ED besteht Therapiebedarf. $\mathrm{Zu}$ den Optionen gehört die Schwellkörperautoinjektion (SKAT), die transurethrale Applikation von Prostaglandin E1 (MUSE) und die Vakuumpumpe.

Mit weitem Abstand die beste Akzep$\tan z$ besteht bei den mittlerweile drei verfügbaren Phosphodiesterase(PDE)-
5-Hemmern Sildenafil, Tadalafil und Vardenafil. Sexuelle Lust vorausgesetzt, unterstützen diese Substanzen die Erektion mit Ansprechraten von $60-80 \%$ bei geringem Nebenwirkungsrisiko.

\section{Ejaculatio praecox oft Anlass für depressive Verstimmungen}

Als mögliche Ursache für Ejakulationsstörungen nannte Humke eine sexuelle Tabuisierung in der Kindheit oder Kastrationsangst. Ebenfalls infrage kommen eine Hypersensitivität der Glans oder eine Serotonin-Rezeptor-Dysfunktion.

Als sekundäre Sexualstörung ist die Ejaculatio praecox oftmals mit einer ED, einer Prostatitis oder einer Schilddrüsenerkrankung assoziiert. Sie belastet den Patienten und die Partnerin, sie führt zu Frust, Isolation, depressiver Verstimmung und Orgasmusstörungen.

Der Trend geht zur medikamentösen Behandlung, z. B. mit dem SerotoninReuptake-Inhibitor Dapoxetin, der die intravaginale Ejakulations-Latenzzeit (IELT) um das Dreifache erhöht. PDE5-Hemmer sind in diesem Zusammenhang nicht sinnvoll, erklärte Humke.

Abschließend unterstrich Humke die Bedeutung der Interdisziplinarität bei der Diagnostik und Therapie von sexuellen Funktionsstörungen. Eine Schlüsselstellung haben Hausärzte, Urologen und Andrologen. Im Einzelfall sollten auch Endokrinologen und Psychologen bzw. Psychotherapeuten hinzugezogen werden. MARTIN BISCHOFF .

- Symposium "Sexuelle Funktionsstörungen“, Medizin 2011, Stuttgart, 28.-30. Januar 2011 (Veranstalter: Bezirksärztekammer Nordwürttemberg) 